Chapter 26

\title{
Basic and Advanced Operative Techniques in Orthognathic Surgery
}

\author{
F. Arcuri, M. Giarda, L. Stellin, A. Gatti, M. Nicolotti, \\ M. Brucoli, A. Benech and P. Boffano \\ Additional information is available at the end of the chapter
}

http://dx.doi.org/10.5772/51762

\section{Introduction}

Orthognathic surgical procedures have been developed to reposition the jaws and have been traditionally used in the dentate patient to correct a skeletal malocclusion; these procedures are usually carried out with orthodontic control of the dentition to produce the best results. The majority of the clinical cases of maxillary deformities can be solved by three basic osteotomies: the LeFort I type maxillary osteotomy (LFI), the bilateral sagittal split osteotomy of the mandible (BSSO) and the horizontal sliding osteotomy of the mandibular symphysis (genioplasty).The LeFort I osteotomy, as described by Obwegeser in 1965, manages the midface; it can be performed as a single-piece monobloc technique or it can be executed as a multisegment procedure or with a distraction approach such as SARPE (Surgically Assisted Rapid Palatal Expansion). The BSSO and the genioplasty, described by the same author in 1955 and in 1957, respectively, allows the surgeon to modify the mandible.[1-3]

Orthognathic surgery can require the execution of codified subapical osteotomies to manage peculiar dento-alveolar discrepancies such as: the segmental anterior maxillary osteotomy according to Wassmund, the segmental posterior maxillary osteotomy according to Schuchardt and the segmental anterior mandibular osteotomy according to Köle.[4-6]Moreover, there are osteotomy well described in the scientific literature but now rarely used in the common practice such as: the intraoral vertical subcondylar osteotomy (Hebert, 1970), the median mandibular osteotomy, the maxillary-zygomatic osteotomy and the quadrangular Le Fort I osteotomy.[7-9]Historically, orthognathic surgery is used to correct dento-facial malocclusion and it is a common practice in maxillo-facial surgery; however, based on an extensive review associated with our experience, we report peculiar clinical scenarios, different from simple malocclusion, where orthognathic surgery is a precious tool. 


\section{Obstructive sleep apnea syndrome}

Continuous positive airway pressure therapy (CPAP) is the first line treatment for patients affected by Obstructive Sleep Apnea Syndrome (OSAS). CPAP prevents upper airway collapse, relieves symptoms such daytime sleepiness and decreases the cardiovascular accidents events. However, this treatment has poor patient compliance. An alternative approach to CPAP is upper airway surgery. The goal of surgery is to increase the posterior airway space and decrease the resistance to airflow, removing the site or sites of upper airway collapse.

Different surgical approaches have been proposed in the literature: tracheostomy, uvulopalatopharyngoplasty, hyoid suspension, partial glossectomy, lingual suspension, tongue base resection, genioglossus advancement and maxillomandibular advancement (MMA). Scientific literature considers MMA as the most effective surgical treatment for the management of adult OSAS. Surgical success and long-term stability confirms the efficacy and safety of this procedure. Tracheostomy is the surgical treatment for OSA patients with a success of $100 \%$ because it bypasses the site of collapse; however, it is indicated as a treatment of last resort after the failure of other surgical procedures. The reported surgical success rate for soft tissue surgical procedures is approximately 40-60\%. MMA enlarges the pharyngeal space by expanding the skeletal framework; MMA is currently the most effective surgical treatment for the management of OSAS in adults.

To assess the surgical success and the long term stability both objective and subjective parameters are generally considered before surgery (T0), at 6 months after surgery (T1) and at follow up (T2). Objective examinations are commonly evaluated by upper airway fibroscopy during the Mueller's manoeuvre, by lateral cephalometry and by polysomnography. Subjective examinations can be evaluated by Epwhorth Sleepiness Scale (ESS) questionnaire.

With upper airway endoscopic evaluation performed by flexible fiberoptic endoscope in supine position during the Mueller's manoeuvre, it can be assessed:

1. the localization of collapse (N: Nose, O: Oropharynx, H: Hypopharynx);

2. the pattern of collapse (c: Circular, t: Transversal, AP: Antero-Posterior);

3. the grade of collapse (grade $0,1,2,3,4)$ (NOH classification).[10-12]

With lateral cephalometry, performed on latero-lateral teleradiography by the same operator, it is possible to evaluate the sskeletal relationship by angular measurements (SNA, SNB) and the posterior air space (PAS) between the base of the tongue and the posterior wall of the pharynx. With polysomnography it can be possible to evaluate the average number of apneas and hypopneas per hour during sleep (AHI), the average number of oxyhemoglobin desaturation per hour during sleep (ODI) and the average time spent with oxyhemoglobin saturation below $90 \%$ during sleep $\left(\mathrm{SaO}_{2}<90\right)$.

Results of OSA surgical treatment are divided into "surgical success" and "surgical cure". Surgical success is defined as an AHI $<20$ events/hour. Surgical cure is defined as an AHI $<5$ events/hour after surgical procedure. Holty and Guilleminault performed a meta-analysis 
regarding the clinical efficacy of MMA in treating OSAS. Six hundred twenty- seven adults with OSAS underwent to MMA. The mean AHI decreased from 63.9 events/h to 9.5 events/h following surgery. The surgical success and cure rates were $86 \pm 30.9 \%$ and $43.2 \pm 11.7 \%$ respectively. Also they observed the maintenance of surgical success rate at 44 months after surgery.[13, 14]

The analysis of skeletal cephalometric values (SNA and SNB) at T1 and at T2 does not show generally significant differences, confirming the long-term stability of skeletal advancement. According to the literature, the postoperative PAS (T1) has commonly an increase. At T2 the PAS maintains stable values. The skeletal advancement is commonly $1 \mathrm{~cm}$ for each jaw. Lye et al. found a statistically significant correlation between the degree of maxillary advancement and reduction in AHI. However, others have reported no association between the degree of maxillary advancement and improvement in AHI after MMA. MMA is generally safe with a reported major surgical complication maxillary (ischemic necrosis, cardiac complication) rate of $1 \%$, minor complication (mandibular relapse, facial paresthesia, temporomandibular joint disorder) rate of $3.1 \%$ and no reported deaths.

OSAS is a chronic disease, so the treatment goal is the control of the symptoms and the control of OSAS-related risks by reducing the severity of the disorder. Surgical success and long term stability of outcomes confirm the efficacy and safety of MMA for treatment of OSAS. However a continuous follow up of these patients is necessary to control their lifestyle and to detect any possible relapse.[15] (Fig. 1 a-d)

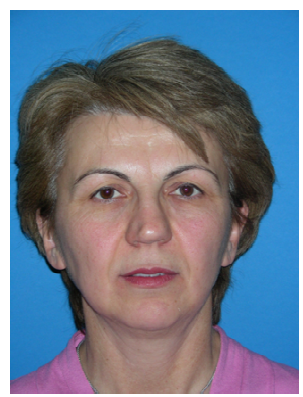

(a)

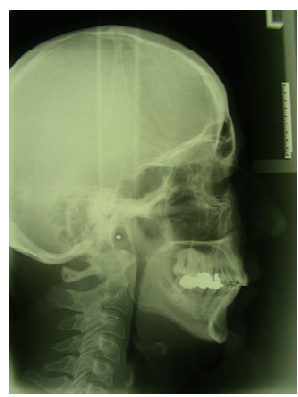

(b)

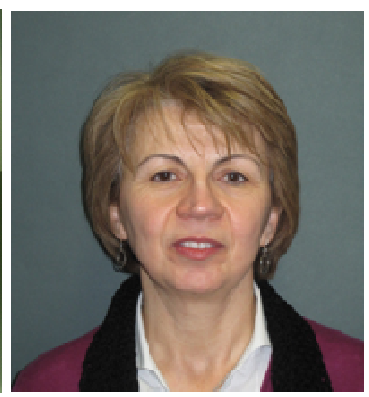

(c)

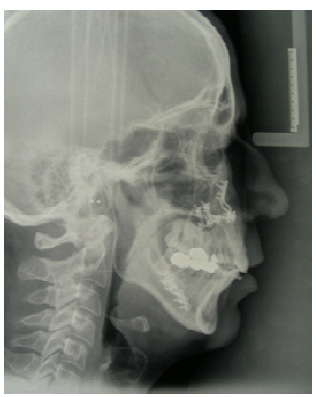

(d)

Figure 1. a) Preoperative frontal view. b) Preoperative radiographic examination. c) Photograph after bimaxillary surgery for advancement. d) Postoperative radiograph demonstrating successful advancement.

\section{Preprosthetic technique in orthognathic surgery}

Orthognathic surgery can be performed on the edentulous patient to correct discrepancies between the jaws, followed by the placement of implants to rehabilitate the maxillary bones; different surgical approaches and technical variations have been proposed. 
This reconstructive method has the advantages over other commonly used preprosthetic techniques of simultaneously allowing the placement of osseointegrated implants, while correcting an unfavourable intermaxillary relationship and improving facial esthetics. [16-18]

Since the 1970s osseointegrated implants have played an important role in oral and maxillofacial reconstruction. Although the success of this method for edentulous jaws with sufficient bone height, patients with an atrophic maxilla and mandible continue to be difficult cases for an optimal outcome in terms of esthetics and function. This condition is characterized by the lack of bone for implants and a reverse maxillomandibular relationship; the progressive loss of alveolar bone height leads to less volume available for the implants with a high rate of surgical failure. Vertically directed resorption increases the interarch space; the projection of the maxilla diminishes in the sagittal plane with change of the intermaxillary relationships and a pseudoprognathism. The combination of loss of projection and diminished vertical bone height results in collapse of the soft tissues of the midface resulting in a more aged face.

Orthognathic surgical procedures have been initially described to reposition the jaws and have been traditionally used in the dentate patient to correct a skeletal malocclusion; these procedures are usually carried out with orthodontic control. Moreover, these procedures are used on the edentulous patient to correct the discrepancies between the maxilla and the mandible associated with the placement of implants to rehabilitate the oral cavity.[19-21] This reconstructive method has the advantages over other commonly used preprosthetic techniques of simultaneously allowing the placement of osseointegrated implants while correcting an unfavourable intermaxillary relationship and reversing facial aging. However, LeFort I osteotomy as a preprosthetic procedure for the atrophic edentulous maxilla is a technically demanding procedure and there are some complications such as infection, hemorrhage, aseptic and avascular necrosis, fractures of the maxilla, bone exposure and oroantral fistulas.

LeFort I osteotomy with interpositional and onlay bone grafts followed by implants' placement is one of the most common methods to manage a deficient vertical and horizontal maxillary dimension. However, this is a two-step procedure involving significant surgery with considerable morbidity at the donor site with a high rate of bone graft resorption. Recently surgeons use a computer-assisted software, which enables them to insert implants after a digital analysis of the residual alveolar and basal bone. This method offers surgeons the possibility of visualizing anatomic structures, evaluating implant position and inclination and to accurately insert implants. Implant-prosthetic rehabilitation can be difficult and affords both functional and psychological improvement. Computer assisted surgery can be the treatment of choice for these conditions; and the insertion of implants in the presence of marked bony defects can be simplified (Fig. 2 a-g).[22, 23] 


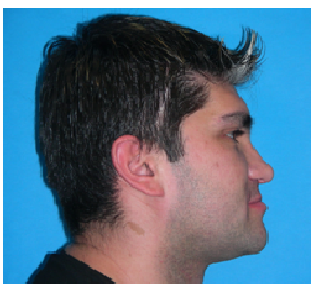

(a)

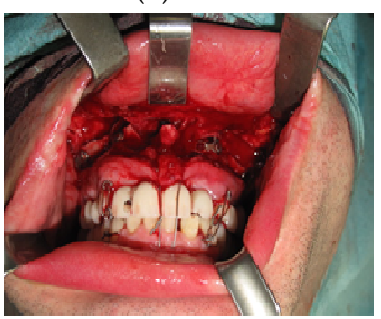

(d)

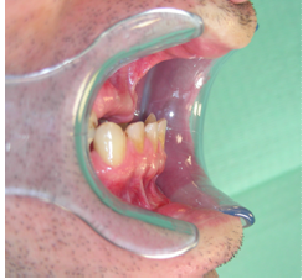

(b)

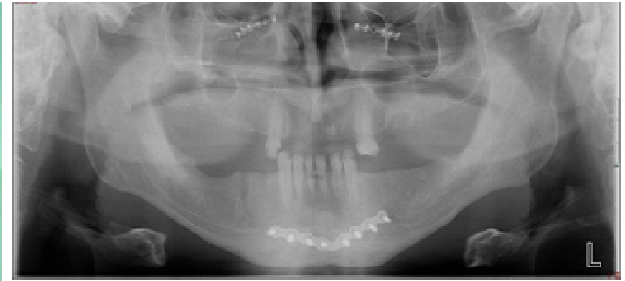

(c)

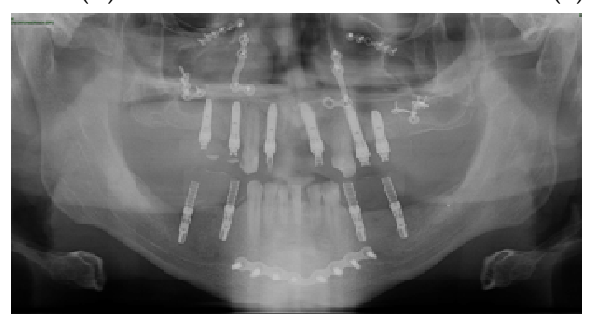

(e)

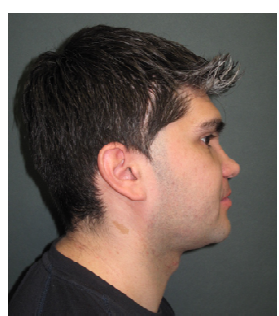

(f)

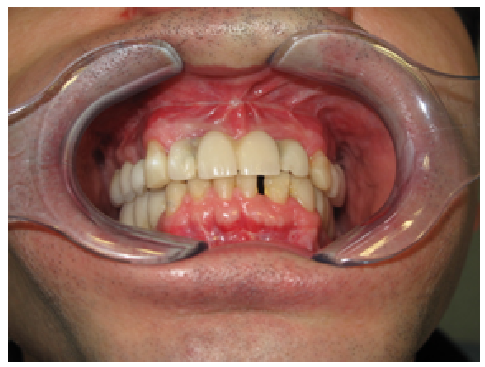

(g)

Figure 2. a) Clinical examination demonstrating a prognathic and reverse maxillary relationship. b) Clinical view revealing partial edentulism of the maxillary and mandibular arches with a severe atrophy of the upper jaw. c) Preoperative orthopantomograph showing the osteosynthesis plates after craniofacial trauma. d) Photograph demonstrating a sequence of the LeFort 1 osteotomy. e) Postoperative radiographic examination showing the adequate osteosynthesis after LeFort I osteotomy and dental implants. f) Postoperative lateral view demonstrating an adequate morphology. g) Occlusal view showing an optimal healing of the intraoral tissues.

\section{Post-traumatic malocclusion}

Facial fractures must be reduced as soon as possible to ensure a proper result; despite a careful surgical technique skeletal and soft-tissue deformities can persist. Orbito-zygomatic, nasal and occlusion problems can occur and result in an unsatisfactory outcome. Orthognathic surgery can be used to manage dentofacial post-traumatic deformities, coordinated with orthodontic and prosthodontic techniques. Management follows the basic rules for correcting primary malocclusion such as: preoperative detailed analysis with clinical records and cephalometric evaluation, well-established orthognathic surgical procedures and postoperative care. 
Post-traumatic malocclusion can occur as a result in delayed treatment for unfavourable clinical conditions of the patient such as neurological, abdominal and thoracic injuries; otherwise it can be the squeal of a bad surgical outcome after a primary surgical treatment. Although post-traumatic deformities of the midface are managed with osteotomy in the lines of fracture such as in the malpositioned zygoma, orthognathic surgery, along with preoperative and postoperative orthodontic treatment, reposition the maxilla and the mandible in the preoperative three-dimensional position. Unsatisfactory outcomes of primary management of complex midfacial fractures can result in displacement of the jaws in the three planes of the space, resulting in altered dental and skeletal relationships.

According to the basic rules of orthognathic surgery, LeFort I single or multisegmental osteotomy and bilateral sagittal split osteotomy are indicated, eventually with bone grafts to support the movement of the jaws in the sagittal, vertical, and transverse planes. Treatment planning include endodontics assessment, orthodontic therapy, prosthodontic rehabilitation. Preoperative records such as dental casts, clinical photographs and radiographs, should be obtained to guarantee a satisfactory result. Mandibular or maxillary non-union is commonly managed with debridement of the original fracture with realignment of the occlusion, autologous bone grafting and osteosynthesis with miniplates and screws.

Post-traumatic maxillary deformities after LeFort fractures show midface retrusion, low facial height, anterior open bite, and mandibular overclosure for posterior displacement of the maxilla; moreover anterior cephalic telescoping of the mandible can be found from inferior pull of the pterygoid musculature on the pterygoid plates. LeFort I osteotomy to correct the malocclusion is often the easiest solution, regardless of the primary fracture. Moreover, if occlusal correction is planned, attention to the transverse dentoalveolar relationships should be addressed to determine if maxillary segmental osteotomies are required or preoperative orthodontic therapy is needed.[24]

Conversely, the most common fracture of the mandible which leads to post-traumatic malocclusion is related to the condyle. Discussion about the primary indication for surgery or closed treatment both in children and in adult patients is beyond the scope of this chapter. However, post-traumatic malocclusion with asymmetry caused by unilateral condylar process fractures can be managed with an osteotomy on the affected side or sometimes on both sides. A symmetric anterior open bite caused by bilateral condylar process fractures presents a surgical dilemma. It can be corrected with maxillary and/or mandibular osteotomies, according to dental, skeletal and esthetics issues. Finally, masticatory dysfunction is primarily related to the post-traumatic malocclusion. However, diminished mandibular movement can also lead to oral dysfunction. Trismus may be the result of temporomandibular joint (TMJ) dysfunction. TMJ dysfunction needs to be managed by a variety of techniques such as: occlusal splints, physiotherapy, and surgical procedures of the TMJ.[25] (Fig. 3 a-d) 


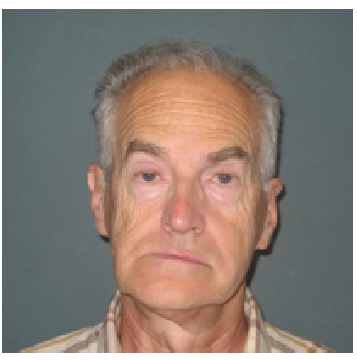

(a)

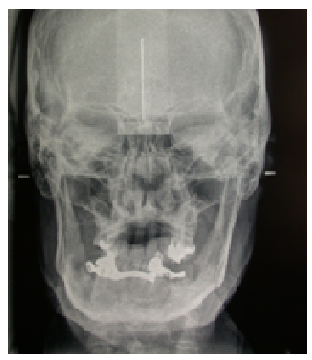

(b)

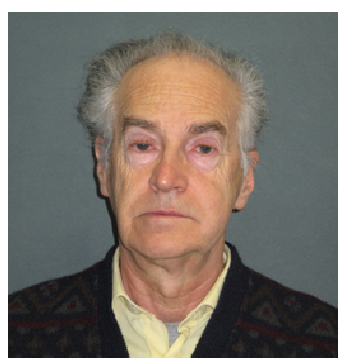

(c)

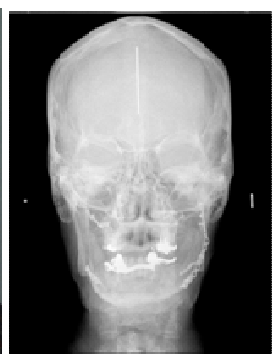

(d)

Figure 3. a) Clinical view of a post-traumatic mandibular laterodeviation. b) Radiographic evaluation. c) Postoperative condition showing facial balance. d) Postoperative control radiograph.

\section{Maxillofacial approach}

There are peculiar clinical circumstances where orthognathic surgery can solve the problem. Extraction of deeply located impacted inferior wisdom molars in close relationship with the inferior alveolar nerve (IAN)[26] or large cysts can be successfully removed by a bilateral sagittal split osteotomy (BSSO) of the mandible.[27]

Although enucleation and/or curettage together with bone removal is the treatment of choice for deeply located mandibular cysts, BSSO can be considered as a valid alternative to the conventional surgical approaches to achieve an adequate exposure of the region of the angle. In this region, the bone between the nerve and the external cortex is thick; therefore, bone removal by a buccal approach can be troublesome, increasing the risk of nerve injury.

The same discussion can be addressed for deeply located wisdom molars where the standard buccal approach poses an unacceptable risk to damage the IAN with excessive bone removal. However, although BSSO guarantees a wide exposure of the IAN, the dental roots and the cystic wall, it is still associated with complications such as: neurosensory disturbances, nonunion, malocclusion, unfavourable fractures, infections and hemorrhage.

Moreover, the mandibular osteotomy can be used as a decompressive technique in case of endodontic overfilling involving the mandibular canal with a potential risk of permanent IAN's injury.[28] Iatrogenic injury to the IAN after endodontic treatment of the posterior mandibular teeth is a well described complication which may lead to sensory disturbances such as pain, hypoesthesia, paresthesia, and dysesthesia of the chin and the lower lip. Two mechanisms are involved in the damage of the nerve: the chemical neurotoxicity of the components of the endodontic material and the mechanical pressure of the material injected into the mandibular canal.

Although decortication in association to apicectomy is considered the treatment of choice for removing endodontic paste, BSSO is also an adequate alternative. In the region of the man- 
dibular angle, the bone is thick and the view is poor; then, decortication with apicectomy removes bone, while increasing the risk of nerve injury with a "blind" approach. However, as the degree of nerve injury increases with time, early surgical decompression of the IAN must be performed, regardless of the surgical approach.

LeFort I osteotomy and its variations are extensively used to approach nasal, paranasal and skull base regions. The removal of cranio-cervical lesions from the sphenoid to the fourth cervical vertebra between the carotids can be relatively easy with the transmaxillary approach.

Lesions that are intrasellar (pituitary tumors, craniopharyngiomas, Rathke's cysts) are frequently approached endoscopically. However, when an extensive exposure is needed, the transmaxillary approach gives a wider access to the clival lesions with superior and inferior extension for both benign neoplasms (angiofibroma, chordoma, fibrous dysplasia meningocele, aneurysm) and malignant tumours (malignant acinic cell, adenocarcinoma, adenoid cystic carcinoma, chondrosarcoma, olfactory neuroblastoma, sarcoma).Complications related to the transmaxillary approach include: injury of the infraorbital nerve, dental roots, tooth buds and lacrimal duct. Moreover avascular and aseptic necrosis of the soft-tissue, bone, and teeth, along with malocclusion, oronasal fistula and velopharyngeal dysfunction are well described.[29]

\section{Clefts and craniofacial syndromes}

Craniofacial morphology of patients affected by lip and palate cleft is characterized by a retrusion of the maxilla. The maxilla shows a various degree of skeletal, soft tissue, and dental deficiency. Maxilla shows clockwise rotation, with an increase of the anterior height of the mandible and a decrease in the posterior height of the maxilla. The severity of the malocclusion and the facial asymmetry indicates the surgical and orthodontic therapy. Surgical procedures performed during childhood are lip and palate clefts reconstruction, alveolar cleft repair and pharyngeal flap. Mild discrepancies of the jaws may be camouflaged by the orthodontic therapy during childhood; however, at the end of the skeletal growth, orthognathic surgery can be the treatment of choice for some cases.[30, 31]

Orthognathic surgery can be performed for the correction of malocclusion in patients with craniofacial syndromes (Crouzon, Apert, Treacher Collins, Hemifacial microsomia, Goldenhar syndrome). Treacher Collins syndrome is characterized by agenesis of the zygomatic bone and hypoplasia of the greater wings of the sphenoid. The zygomatic arch can be absent or hypoplastic; the maxilla and the mandible show a various degree of hypoplasia. Early correction of mandibular defects can be performed with distractors; however, bilateral sagittal split osteotomy (BSSO) and/or LeFort I osteotomy (LFI) at a later age may be needed. LFI addresses the vertical and anterior-posterior defects (open bite); BSSO associated with the horizontal sliding osteotomy of the mandibular symphysis corrects the mandibular defect.

Goldenhar syndrome is a bilateral disease, which is characterized by a degree of agenesis and hypoplasia of the mandibular ramus, mandibular condyle, tragus, helix, antihelix, and 
temporomandibular joint (TMJ). The chin shows a degree of deviation and the margin of the mandible of the affected side is higher than the contralateral. The occlusion is Class II and the lower midline is displaced to the affected side. It should be treated by bilateral sagittal split osteotomy (BSSO) or mandibular osteodistraction based on the degree of severity and the experience of the surgeon. In case of severe deformity such as a serious joint involvement, BSSO may be indicated early around 9 years of age and it can be used with bone grafts for the restoration of the integrity of the ramus. However, the surgical correction of malocclusion occurs mostly in cases at the end of growth.

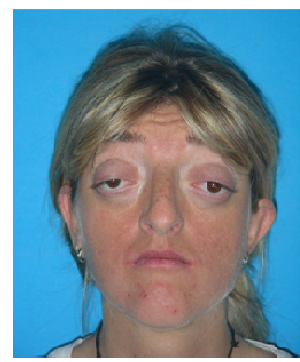

(a)

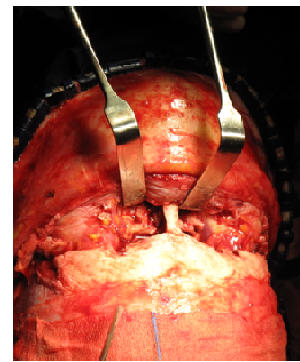

(d)

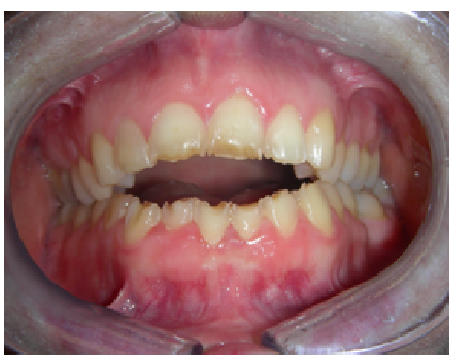

(b)

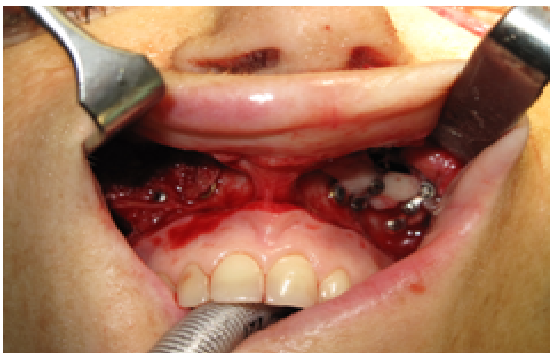

(e)

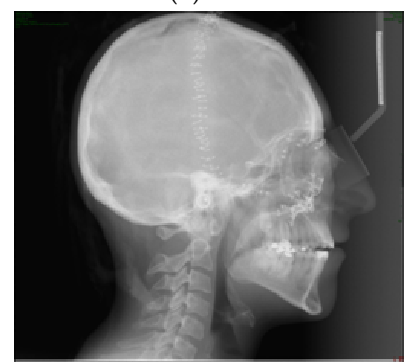

(g)

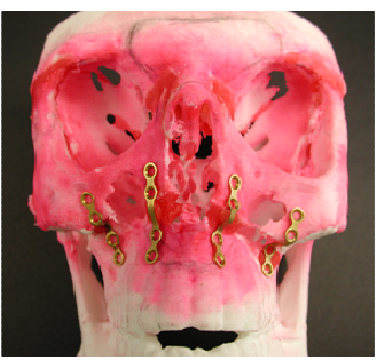

(c)

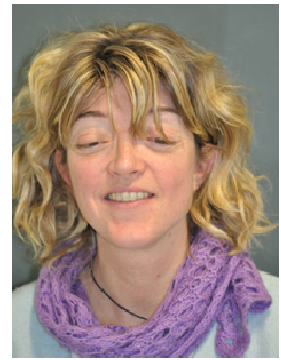

(f) Figure 4. a) Preoperative frontal view showing the craniofacial malformation. b) Occlusal view demonstrating the
open bite. c) Acrylic model with maxillary titanium plates adapted preoperatively to reproduce the LeFort I osteotomy. d) Intraoperative sequence showing the frontonasal surgical procedure. e) Intraoperative view of the LeFort I osteosynthesis. f) Postoperative photograph demonstrating an acceptable result. g) Postoperative radiographic control after surgery. 
Apert and Crouzon syndrome are diseases characterized by synostosis of multiple sutures of the skull and the face; these diseases show a severe maxillary retrusion with a Class III malocclusion and open bite. The mandible has a normal shape. Common features are a narrow/high-arched palate, posterior bilateral crossbite, hypodontia, and crowding of teeth. The treatment begins early in the neonatal period if there are signs and symptoms of increased intracranial pressure. The first procedure is the advancement of the fronto-orbital complex to restore the cranial shape. The second step begins at around 6 years of age. The facial complex is osteotomized according to the Le Fort III line, eventually with a median osteotomy creating a facial bipartition. At the end of growth in many patients there is still a malocclusion. Surgical procedures depend on the defects; however, LFI is used to advance the maxilla, while correcting the open bite.[32] (Fig. 4 a-g)

\section{Reverse facelift}

The physiopathological basis of the aging face is not completely understood; however three factors contribute to the development of the aforementioned problem: soft tissue laxity, soft tissue atrophy and skeletal resorption. The aging face is characterized by multiple signs affecting the upper third (brow ptosis, excess of upper eyelid skin, forehead furrows, herniation of the orbital fat pad, glabellar frown lines); the middle third (accentuation of the parabuccal fat pad and development of the nasojugal fold) and the lower third (evidence of the labiomental fold, formation of the facial jowls and accentuation of the submental fat pad).[33-36]

Facelift procedures and fat grafting have been developed to restore a younger face and address the laxity and the atrophy of the soft tissue; the classic concept is that during life the force of gravity pulls the facial teguments down; facelift procedures pull the tissues up, both conventionally and more recently endoscopically. Moreover structural fat grafting accentuates the atrophic facial soft tissue and recreates the lost young tension.[37-39]

It is a common belief that the maxillofacial skeleton atrophies with the aging process, leading to a reduction of the facial height and depth; maxillary and mandibular bone resorption leads to a loss of support of the mouth and the nose. Maxillomandibular advancement (MMA) by orthognathic surgery restores the lost space dimension, providing projection to the cheeks, the jaws and the nose. In relation to the satisfactory esthetic results of orthognathic procedures performed on OSAS patients, the concept of "reverse face lift" started to arise. Maxillomandibular advancement is a very powerful tool to mask the physiological bone atrophy. It restores the space dimension by projecting the nose, the cheeks and the mouth.

The effect of bimaxillary manipulation on the facial soft tissue for dentofacial deformities has long been studied; conversely, the resultant facial changes of patients treated by MMA for OSAS has not been adequately described and the concept of "reverse face lift" has not been investigated in the scientific literature. Simultaneously MMA changes the skeletal framework of the face, improving soft tissue support and resulting in rejuvenation of the middle and the lower third of the face. 


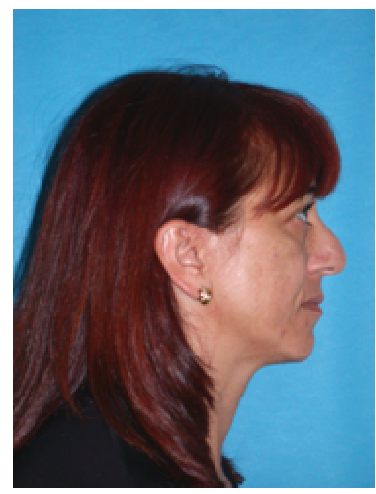

(a)

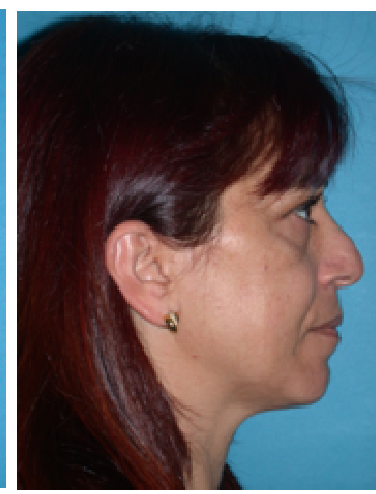

(b)

Figure 5. a) Lateral photograph of an aging face. b) Postoperative view after bimaxillary surgery of advancement showing the effects of reverse facelift.

Preoperative analysis of facial proportions with cephalometric measures, as performed with standard orthognathic cases, is of paramount importance before performing MMA for OSAS. Eventual unesthetic facial changes must be preoperatively discussed with the patient and the necessity of clockwise/counterclockwise rotation of the occlusal plane needs to be assessed in order to obtain a satisfactory result in terms of esthetics and functionality. Reverse facelift via bimaxillary advancements is a surgical procedure that may be combined with facelift procedures and structural fat grafting, can be indicated for a selected group of middle-aged patients, very motivated to an extreme rejuvenation. (Fig. 5a, b) [40-43]

\section{Transgender surgery}

Transsexualism is the extreme side of a wide spectrum of disorders called gender identity disorder (GID). It occurs when the anatomic gender of a person is opposite of his or her psychological gender. Epidemiological studies in the United States and Great Britain declare a prevalence of transsexualism of 1:50,000.

There are essential differences between male and female faces with regard to the skeleton and the soft tissues of the face. They have been extensively studied. The male forehead is flat and the supraorbital ridges are prominent. Females have a higher forehead with a more convex curvature. The orbits of the women are larger and higher. The zygomas of men are larger but less prominent. The mandible of men is larger, with a more prominent gonial angle and a rectangular chin.[44-46]

Gender reassignment requires both medical and surgical treatments. Hormonal therapy must be initiated early in the transgender process in order to change the physical features. The need for facial surgery to pass as a member of the other sex occurs in a significant percentage of transsexuals. 
Facial feminization surgery (FFS) is referred as a group of surgical techniques devoted to change the features of a face from male to female. FFS was pioneered by Dr. Douglas Ousterhout from San Francisco, CA, USA in the 1980s. Facial feminization surgery (FFS) occurs more frequently than facial skeletal masculinisation and it is considered technically less demanding. Orthognathic surgery is a precious tool for a facial sexual reassignment surgical program.[47-50]Maxillary and mandibular osteotomies with clockwise rotation of the bimaxillary complex decreases the projection of both the chin and the mandibular angle region. Preoperative and postoperative orthodontic treatment is of paramount importance for the treatment plan. Le Fort I osteotomy (LFI) in association to a bilateral sagittal split osteotomy (BSSO) changes the geometry of the maxillo-mandibular complex.

The upper jaw can be placed forward in combination with a posterior vertical impaction. Although the mandibular angle does not change position with the BSSO and the dental occlusion remains unchanged, this clockwise rotation of the lower half of the face results in a more convex profile of the face with a less prominent chin which lead to a more feminine facial skeleton.

Orthognathic surgery is frequently associated with other procedures such as:

1. Mandibular angle reshaping to reduce the lower facial width;

2. Chin reduction to reshape the stigmata of the masculine chin;

3. Zygoma osteotomies with or without autogenous grafts/alloplastic implants to increase the mid-facial prominence;

4. Forehead recontouring to eliminate the frontal bossing;

5. Rhinoplasty to correct the stigmata of the male midface;

6. Browlift and scalp advancement to feminize the upper third of the face;

7. Lip lift, fat grafting and thyroid cartilage shave as ancillary procedures.[51] (Fig. 6 a-f)

\section{Ethnic orthognathic surgery}

There are certain differences in dental, skeletal, and soft-tissue facial morphology between Afro-American, Asian, Caucasian and Latin patients; orthognathic surgery must be adapted to each peculiar ethnic case. Meticulous planning ad careful execution of the osteotomies according to the preoperative surgical plan is essential to ensure an optimal outcome. Ethnic differences are related to the shape and the proportions of the skeletal framework, the soft tissue, and the texture of the skin. Individuals of all races, all over the world, desire to have an estheticallyideal face. It is essential to understand the ethnic concepts of beauty for an optimal result.

Latin patients descend from the European immigrants and from the native population. For historical reasons, there is a Mongoloid component in their facial shape, making the same criteria of maxillofacial surgery applicable even for Asian populations. Because of similarities in anatomic characteristics such as skin thickness, wide bigonial angle and bimaxillary 


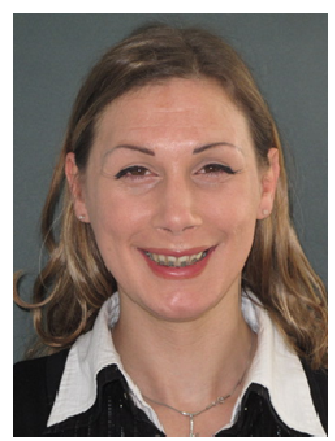

(a)

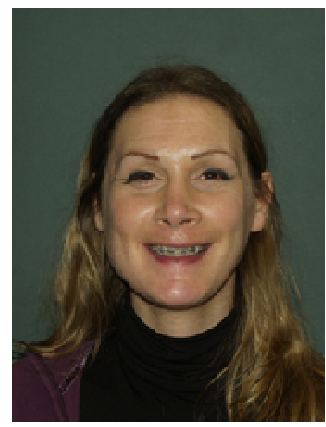

(d)

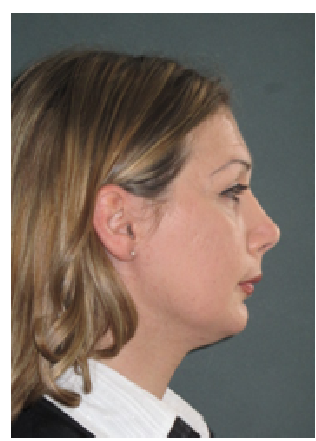

(b)

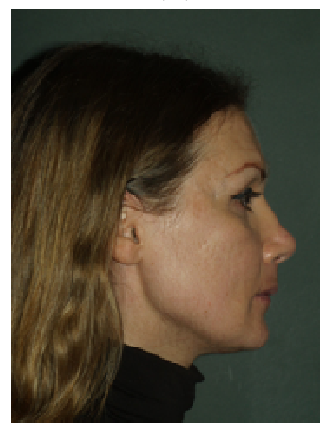

(e)

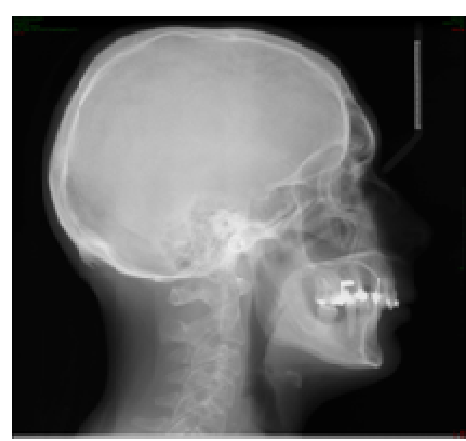

(c)

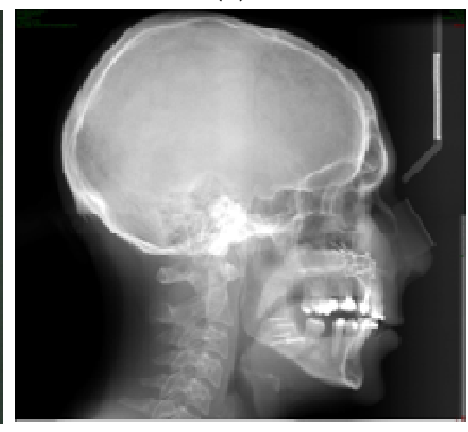

(f)

Figure 6. a) Preoperative frontal view. b) Preoperative lateral view. c) Preoperative radiographic examination. d) Postoperative smile after bimaxillary surgery. e) Lateral photograph demonstrating a feminine appearance. f) Teleradiography demonstrating orthognathic surgery.

protrusion, basic concepts can be applied even for some individuals of African origin. [52]A common characteristic is the protrusion of the dental arches, which lead to the projection of the lips with an acute nasolabial angle and the absence of the sublabial sulcus. The gingival display is excessive and the lip strain is exaggerated; the nasal spine appears receded and the paranasal areas appear depressed. The chin is located in a normal position; however it frequently appears receded because of the prominence of the dental arches; this feature augments the facial convexity. Standard surgical procedures include: Lefort I osteotomy to correct the midfacial deformities, bilateral sagittal split osteotomy to adapt the mandible, and subapical osteotomies to manage peculiar dento-alveolar discrepancies.

Surgical approach to alveolar protrusion requires careful planning and preoperative orthodontics. Model surgery needs to be performed in order to coordinate the dental arches after segmental surgery; finally intraoperative occlusal plates are fabricated. Two splints are necessary if bimaxillary protrusion is managed in a single stage as double-jaw surgery. Sophisticated studies about the vascularity of the maxilla and surgical refinements regarding the osteotomies lines have guaranteed predictable outcomes with minimal morbidity. Segmen- 
tal osteotomies need to be performed without injuring adjacent teeth, while preserving the blood supply from the mucosa to the osseous segments.

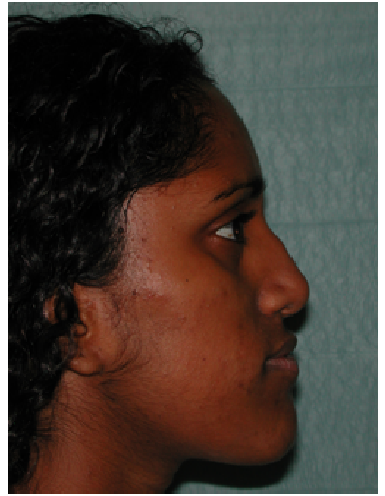

(a)

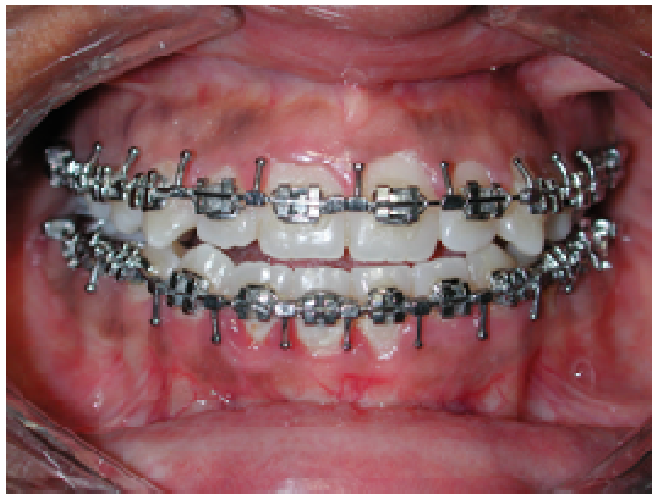

(b)

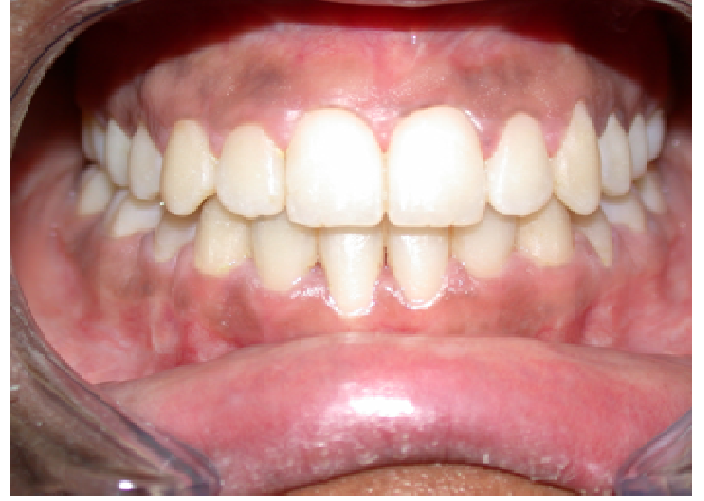

(c)

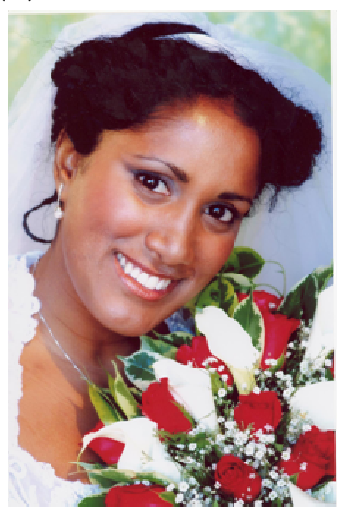

(d)

Figure 7. a) Preoperative frontal view. b) Preoperative malocclusion III class. c) Postoperative smile demonstrating a satisfactory result. d) Postoperative occlusal view.

The procedure can be managed by general or local anaesthesia. A vertical incision is performed on each side of the upper arch from the alveolus of the first premolar, which is extracted, toward to the vestibular sulcus. A segment of bone is removed from the palatine process and from the alveolar arch in order to displace the premaxilla backward. Osteosynthesis is performed with titanium plates and screws eventually associated to orthodontic bar.

Deformity of the mandibular dental arch is managed in a similar fashion. The incision is placed vertically in the mucosa from the first premolar toward the vestibular cul-de-sac. Then, subperiosteal dissection of the buccal and lingual cortex of the mandible is executed. One vertical osteotomy for each side of the arch is extended beyond the dental roots. Then, a 
horizontal osteotomy is made joining the aforementioned osteotomies. The excess of bone is resected. The segment is mobilized and with the occlusal bite in place osteosynthesis is done with plates and screws.

Orthognathic procedures for correcting skeletal deformities can be used in association with maxillary and mandibular osteotomies. Frequently, skeletal surgery is combined with adjunctive procedures such as: forehead lift, facelift, rhinoplasty and fat grafting to augment facial beauty.[53,54] (Fig. 7a-d)

\section{Reoperative orthognathic surgery}

Although orthognathic surgery is considered a routine procedure in the common practice of oral and maxillofacial surgery, problems can arise at any point of the orthodontic-surgical process: the preoperative diagnosis and planning, the orthodontic therapy and the surgical phase. Complications can be divided into: airway, vascular, neurologic, infectious, dental, skeletal and cosmetic. Complications which require reoperation can occur; problems must be careful identified and solved to obtain an optimal result in terms of esthetics and functionality.

A full description based on an extensive literature review regarding the incidence of the complications among the different orthognathic procedures is beyond the scope of this chapter. However, intraoperative and/or postoperative hemorrhage, hypoesthesia /anaesthesia of the trigeminal branches, lesion of the cranial nerves and the skull base, maxillary avascular and aseptic necrosis and bone or soft tissue infection can occur at any time even for the most experienced surgeon.

Reoperative orthognathic surgery is required when the results obtained after the initial treatment are not satisfactory in terms of esthetics or functionality. Complications which require reoperation can occur during the surgery, in the initial postoperative phase, and after weeks/ months from the initial treatment.

The proper position of the condyle in the glenoid fossa is a manoeuvre which tremendously affects the final dental and skeletal occlusion. Condylar sag can be classified as central, peripheral type I and peripheral type II from maxillary or mandibular surgery. Central condylar sag can occur if the condyle is positioned inferiorly in the glenoid fossa without bone contact with the fossa. After removal of the intraoperative maxillo-mandibular fixation (IMMF), the condyle will move superiorly, causing an anterior open bite if the problem is bilateral. If only 1 side is affected, the lower dental midline will move toward the affected side and the occlusion of the affected side will be class II.

Peripheral condylar sag type II occurs when excessive pressure is placed on the proximal segment during osteosynthesis which leads to a superolateral movement of the condyle. If it occurs bilaterally, the final occlusion will be a posterior open bite; if it occurs only on 1 side, the occlusion will be a posterior bite only on the affected side and the lower dental midline will move toward to the opposite side of the affected side.[55] 
Central condylar sag may also occur after Le Fort I osteotomy. Condyles may be inferiorly distracted from the glenoid fossa due to posterior bony interference of the maxilla. When IMMF is applied, the mandible will rotate counter clockwise with the posterior teeth as a fulcrum. When IMMF is removed, a class II anterior open bite can result. This event can occur both intraoperatively or in the immediate postoperative period.

Late postoperative complications which require orthognathic surgery can be due to unexpected postoperative growth, idiopathic condylar resorption or peripheral condylar sagging type I. Unexpected late facial growth may take place months or years after the surgical procedure. This is a very challenging issue for the surgeon to determine if the mandibular growth continues and if it should be treated orthodontically or surgically.

Idiopathic condylar resorption is related to the effects of chronic excessive loading of the mandibular condyle. It affects bilaterally and symmetrically the condyle of women between the age of 15 and 30 years. The resorption is progressive and painless, leading to a gradual loss of the ramus height, with a class II anterior open bite. A technetium 99m bone scan will determine if the bone activity is active. Occlusion should be stable for a minimum of 1 year. Patients can be treated by means of orthognathic surgery or with replacement of the mandibular condyle with a total-joint temporomandibular joint prosthesis in cases of severe functional and esthetic problems.

Peripheral condylar sag type I occurs when excessive pressure is placed on the mandibular condyle during osteosynthesis of the fragments which lead to an inferiorly sliding of the condyle with bone contact. This provides stability to the occlusion, and the problem can not be identified at the time of surgery. Resorption of the lateral pole of the condyle can make the problem become apparent even months after surgery. This resorption will cause the condyle to slide superiorly into the fossa; the mandible will relapse posteriorly on the affected side.

Finally, after 6-12 months after surgery, any unsatisfactory esthetic results are analyzed and corrective surgery can be eventually scheduled for soft tissue problems (nasal, midface, lip esthetics) and hard tissue concerns (facial asymmetry, anteroposterior and vertical discrepancies).[56]

\title{
Author details
}

\author{
F. Arcuri ${ }^{*}$, M. Giarda ${ }^{1}$, L. Stellin ${ }^{1}$, A. Gatti ${ }^{1}$, M. Nicolotti ${ }^{1}$, M. Brucoli ${ }^{1}$, A. Benech ${ }^{1}$ and \\ P. Boffano ${ }^{2}$
}

*Address all correspondence to: fraarcuri@libero.it

1 Department of Maxillo-Facial Surgery, Novara Major Hospital: University of Eastern Piedmont "Amedeo Avogadro", Novara, Italy

2 University of Turin, Italy 


\section{References}

[1] Obwegeser, H. (1965). Surgery of the maxilla for the correction of prognathism. Schweizerische Montatsschrift fur Zahnheilkunde, 75, 365-74.

[2] Obwegeser, H. (1964). The indications for surgical correction of mandibular deformity by the sagittal splitting technique. Br J Oral Surg, 2, 157-71.

[3] Trauner, R., \& Obwegeser, H. (1957). The surgical correction of mandibular prognathism and retrognathia with consideration of genioplasty: Part I. Surgical procedures to correct mandibular prognathism and reshaping of the chin. Oral Surg, 10, 667.

[4] Bell, W.H. (1977). Correction of maxillary excess by anterior maxillary osteotomy. A review of three basic procedures. Oral Surg Oral Med Oral Pathol, 43(3), 323-32.

[5] Lachard, J., Blanc, J. L., Lagier, J. P., Cheynet, F., Le Retraite, C., \& Saban, Y. (1987). Köle's operation. Rev Stomatol Chir Maxillofac, 88(5), 306-10.

[6] Ermel, t., Hoffmann, J., Alfter, G., \& Göz, G. (1999). Long-term stability of treatment results after upper jaw segmented osteotomy according to Schuchardt for correction of anterior open bite. J Orofac Orthop, 60(4), 236-45.

[7] Hebert, J. M., Kent, J. N., \& Hinds, E. C. (1970). Correction of prognathism by intraoral vertical subcondylar osteotomy. J Oral Surg, 28, 651-665.

[8] Abubaker, A. O., \& Sotereanos, G. C. (1991). Modified Le Fort I (Maxillary-Zygomatic) osteotomy: rationale, basis, and surgical technique. J Oral Maxillofac Surg, 49, 1089-1097.

[9] Keller, E.E, \& Sather, A.H. (1990). Qadrangular Le Fort I osteotomy: surgical technique and review of 54 patients. J Oral Maxillofac Surg, 48, 2-11.

[10] Mc Ardle, N., Devereux, G., et al. (1999). Long term use of CPAP therapy for sleep apnea/hypopnea syndrome. Am J Respir Crit Care Med, 159, 1108-14.

[11] Lin, H. C., Friedman, M., Chang, H. W., \& Gurpinar, B. (2008). The efficacy of multilevel surgery of upper airway in adults with obstructive sleep apnea/hypopnea syndrome. Laryngoscope, 118, 902-908.

[12] Fairburn, S. C., Waite, P. D., Vilos, G., et al. (2007). Three-dimensional changes in upper airways f patients with obstructive sleep apnea following maxillomandibular advancement. J Oral Maxillofac Surg, 65, 6-12.

[13] Lye, K. W., Waite, P. D., Meara, D., \& Wang, D. (2008). Quality of life evaluation of maxillo-mandibular advancement surgery for treatment of obstructive sleep apnea. J Oral Maxillofac Surg, 66, 968-972. 
[14] Holty, J. C., \& Guilleminault, C. (2010). Maxillomandibular advancement for treatment of obstructive sleep apnea: a systematic review and meta analysis. Sleep Med Rev, 14, 287-297.

[15] Giarda, M., Brucoli, M., Arcuri, F., Braghiroli, A., Aluffi, P., \& Benech, A. (2012). Proposal of a presurgical algorithm for patients affected by obstructive sleep apnea syndrome. J Oral Maxillofac Surg, Jan 27, Epub ahead of print.

[16] Branemark, P. I., Hansson, B. O., Adell, R., et al. (1977). Osseointegrated implants in the treatment of the edentulous jaw. Experience from a 10-year period. Scand J Plast Reconstr Surg Suppl, 16, 1-132.

[17] Albrektsson, T. (1988). A multicenter report on osseointegrated oral implants. J Prosthet Dent, 60, 75-84.

[18] Adell, R., Lekbolm, U., Rockier, B., et al. (1981 A). A 15-year study of osseointegrated implants in the treatment of the edentulous jaw. Int J Oral Surg, 10, 387-416.

[19] Sailer, H.F. (1989). A new method of inserting endosseous implants in totally atrophic maxillae. J Craniomaxillofac Surg, 30, 299-305.

[20] Cawood, J. I., \& Stoelinga, P. J. (2000). International Research Group on Reconstructive Preprosthetic Surgery. Consensus report. Int J Oral maxillofac Surg, 29, 159-162.

[21] Malo, P., de Araujo, Nobre. M., \& Lopes, A. (2007). The use of computer-guided flapless implant surgery and four implants placed in immediate function to support a fixed denture: preliminary results after a mean follow-up period of thirteen months. J Prosthet Dent, 97, S26-S34.

[22] Johansson, B., Friberg, B., \& Nilson, H. (2009). Digitally planned, immediately loaded dental implants with prefabricated prostheses in the reconstruction of edentulous maxillae: a 1-year prospective, multicenter study. Clin Implant Dent Relat Res, 11, 194-200.

[23] Yerit, K. C., Martin, P., Guserl, U., Turhani, D., Schopper, C., Wanschitz, F., Wagner, A., Watzinger, F., \& Ewers, R. (2004). Rehabilitation of the severely atrophied maxilla by horseshoe Le Fort I osteotomy (HLFO). Oral Surg Oral Med Oral Pathol Oral Radiol Endod, 97, 683-692.

[24] Yang, R. S., Salama, A. R., \& Caccamese, J. F. (2011). Reoperative midface trauma. Oral Maxillofac Surg Clin North Am, 23(1), 31-45.

[25] Imola, MJ, Ducic, Y., \& Adelson, R. T. (2008). The secondary correction of post-traumatic craniofacial deformities. Otolaryngol Head Neck Surg, 139(5), 654-60.

[26] Jones, T. A., Garg, T., \& Monaghan, A. (2004). Removal of a deeply impacted mandibular third molar through a sagittal split ramus osteotomy approach. $\mathrm{Br} \mathrm{J}$ Oral Maxillofac Surg, 42(4), 365-8. 
[27] Scolozzi, P., Lombardi, T., \& Jaques, B. (2007). Le Fort I Type Osteotomy and Mandibular Sagittal Osteotomy as a Surgical Approach for Removal of Jaw Cysts. J Oral Maxillofac Surg, 65, 1419-1426.

[28] Scolozzi, P., Lombardi, T., \& Jaques, B. (2004). Successful inferior alveolar nerve decompression for dysesthesia following endodontic treatment: report of 4 cases treated by mandibular sagittal osteotomy. Oral Surg Oral Med Oral Pathol Oral Radiol Endod, 97(5), 625-31.

[29] Girish, Rao. S., Sudhakara, Reddy. K., \& Sampath, S. (2012). Lefort I access for juvenile nasopharyngeal angiofibroma (JNA): a prospective series of 22 cases. J Craniomaxillofac Surg, 40(2), e 54-8.

[30] Heliövaara, A., Ranta, R., Hukki, J., \& Rintala, A. (2002). Skeletal stability of Le Fort I osteotomy in patients with isolated cleft palate and bilateral cleft lip and palate. Int $J$ Oral Maxillofac Surg, 31(4), 358-63.

[31] Wolford, L. M., Cassano, D. S., Cottrell, D. A., El Deeb, M., Karras, S. C., \& Goncalves, J. R. (2008). Orthognathic surgery in the young cleft patient: preliminary study on subsequent facial growth. J Oral Maxillofac Surg, 66(12), 2524-36.

[32] Kahnberg, K. E., \& Hagberg, C. (2010). Orthognathic surgery in patients with craniofacial syndrome. I. A 5-year overview of combined orthodontic and surgical correction. J Plast Surg Hand Surg, 44(6), 282-8.

[33] Arcuri, F, Brucoli, M, Benech, R, Giarda, M, \& Benech, A. (2011). Maxillomandibular advancement in obstructive sleep apnea syndrome patients: a surgical model to investigate reverse face lift. J Craniofac Surg, 22(6), 2148-52.

[34] Sherris, D. A., \& Larrabee, W. F. Jr. (1996). Anatomic considerations in rhytidectomy. Facial Plast Surg, 12(3), 215-22.

[35] Ramirez, O.M. (2000). The central oval of the face: tridimensional endoscopic rejuvenation. Facial Plast Surg, 16(3), 283-98.

[36] Coleman, S. R. (1998). Structural fat grafting. Aesthet Surg J, 18(5), 386-388.

[37] Arnett, G. W., \& Gunson, M. J. (2010). Esthetic treatment planning for orthognathic surgery. J Clin Orthod, 44(3), 196-200.

[38] Hwang, S. J., Haers, P. E., Seifert, B., \& Sailer, H. F. (2004). Non-surgical risk factors for condylar resorption after orthognathic surgery. J Craniomaxillofac Surg, 32(2), 103-11.

[39] Pessa, J. E., Zadoo, V. P., Mutimer, K. L., Haffner, C., Yuan, C., De Witt, A. I., \& Garza, J. R. (1998). Relative maxillary retrusion as a natural consequence of aging: combining skeletal and soft-tissue changes into an integrated model of midfacial aging. Plast Reconstr Surg, 102(1), 205-12. 
[40] Riley, R. W., Powell, N. B., \& Guilleminault, C. (1993). Obstructive sleep apnea syndrome: A review of 306 consecutively treated surgical patients. Otolaryngol Head Neck Surg, 108, 117-25.

[41] Abramson, Z., Susarla, S., August, M., Troulis, M., \& Kaban, L. (2010). Three-dimensional computed tomographic analysis of airway anatomy in patients with obstructive sleep apnea. J Oral Maxillofac Surg, 68(2), 354-62.

[42] Joss, C. U., Joss-Vassalli, I. M., Bergé, S. J., \& Kuijpers-Jagtman, A. M. (2010). Soft tissue profile changes after bilateral sagittal split osteotomy for mandibular setback: a systematic review. J Oral Maxillofac Surg, 68(11), 2792-801.

[43] Jensen, A. C., Sinclair, P. M., \& Wolford, L. M. (1992). Soft tissue changes associated with double jaw surgery. Am J Orthod, 101, 266.

[44] Shams, M. G., \& Motamedi, M. H. (2009). Case report: feminizing the male face. Eplasty, 9, e2, Epub Jan 9.

[45] Cohen-Kettenis, P. T., \& Gooren, L. J. (1999). Transsexualism: a review of etiology, diagnosis and treatment. J Psychosom Res, 46, 315-33.

[46] Hoenig, J., \& Kenna, J. C. (1974). The prevalence of transsexualism in England and Wales. Br J Psychiatry, 124, 181-90.

[47] Monstrey, S., Hoebeke, P., Dhont, M., et al. (2001). Surgical therapy in transsexual patients: a multidisciplinary approach. Acta Chir Belg, 101, 200-9.

[48] Ousterhout, D.K. (1987). Feminization of the forehead: contour changing to improve female anesthetics. Plast Reconstr Surg, 79, 701.

[49] Mommaerts, M. Y., Abeloos, J. V. S., Calix, A. S., et al. (1995). The "sandwich" zygomatic osteotomy: technique, indications and clinical results. J Craniomaxiliofac Surg, 23, 12-9 .

[50] Ousterhout, D.K. (2011). Dr. Paul Tessier and facial skeletal masculinization. Ann Plast Surg, 67(6), 10-5.

[51] Becking, A. G., Tuinzing, D. B., Hage, J. J., \& Gooren, L. J. (1996). Facial corrections in male to female transsexuals: a preliminary report on 16 patients. J Oral Maxillofac Surg, 54(4), 413-418.

[52] Beugre, J. B., Sonan, N. K., Beugre-Kouassi, A. M., \& Djaha, F. (2007). Comparative cephalometric study of three different ethnic groups of black Africa with normal occlusion. Odontostomatol Trop, 30(117), 34-44.

[53] Chew, M.T. (2006). Spectrum and management of dentofacial deformities in a multiethnic Asian population. Angle Orthod, 76(5), 806-9. 
[54] Clemente-Panichella, D., Suzuki, S., \& Cisneros, G. J. (2000). Soft to hard tissue movement ratios: orthognathic surgery in a Hispanic population. Int J Adult Orthodon Orthognath Surg, 15(4), 255-64.

[55] Reyneke, J.P. (2011). Reoperative orthognathic surgery. Oral Maxillofac Surg Clin North Am, 23(1), 73-92.

[56] Chow, L. K., Singh, B., Chiu, W. K., \& Samman, N. (2007). Prevalence of postoperative complications after orthognathic surgery: a 15 -year review. J Oral Maxillofac Surg, 65(5), 984-92. 
